\title{
Adjustment disorders in primary care: prevalence, recognition and use of services ${ }^{\dagger}$
}

Anna Fernández, Juan M. Mendive, Luis Salvador-Carulla, María Rubio-Valera, Juan Vicente Luciano, Alejandra Pinto-Meza, Josep Maria Haro, Diego J. Palao, Juan A. Bellón, Antoni Serrano-Blanco and the DASMAP investigators

\section{Background}

Within the ICD and DSM review processes there is growing debate on the future classification and status of adjustment disorders, even though evidence on this clinical entity is scant, particularly outside specialised care.

\section{Aims}

To estimate the prevalence of adjustment disorders in primary care; to explore whether there are differences between primary care patients with adjustment disorders and those with other mental disorders; and to describe the recognition and treatment of adjustment disorders by general practitioners (GPS)

\section{Method}

Participants were drawn from a cross-sectional survey of a representative sample of 3815 patients from 77 primary healthcare centres in Catalonia. The prevalence of current adjustment disorders and subtypes were assessed face to face using the Structured Clinical Interview for DSM-IV Axis I Disorders (SCID-I). Multilevel logistic regressions were conducted to assess differences between adjustment disorders and other mental disorders. Recognition and treatment of adjustment disorders by GPs were assessed through a review of patients' computerised clinical histories

\section{Results}

The prevalence of adjustment disorders was $2.94 \%$. Patients with adjustment disorders had higher mental quality-of-life scores than patients with major depressive disorder but lower than patients without mental disorder. Self-perceived stress was also higher in adjustment disorders compared with those with anxiety disorders and those without mental disorder. Recognition of adjustment disorders by GPS was low: only 2 of the 110 cases identified using the SCID-I were detected by the GP. Among those with adjustment disorders, $37 \%$ had at least one psychotropic prescription.

\section{Conclusions}

Adjustment disorder shows a distinct profile as an intermediate category between no mental disorder and affective disorders (depression and anxiety disorders).

\section{Declaration of interest}

None.
With the reviews of the Diagnostic and Statistical Manual of Mental Disorders (DSM) and the International Statistical Classification of Diseases and Related Health Problems (ICD) criteria for their 5th and 11th versions respectively, concerns about the relevance and usefulness of adjustment disorder diagnoses re-entered the mental health arena. ${ }^{1-5}$ There are those who strongly support the value of the concept of adjustment disorder as a category, but are critical of the current criteria because of their vagueness and because they fail to sufficiently distinguish adjustment disorders from normal reactions to stressors. ${ }^{2-4,6,7}$ Despite frequent statements on the high prevalence of adjustment disorders, few studies have addressed this issue. In fact, adjustment disorders have not been included in any of the main epidemiological studies carried out in recent years such as the World Health Organization (WHO) Mental Health Epidemiological Survey, the Epidemiologic Catchment Area study and the National Comorbidity Survey Replication. To the best of our knowledge, only the European Outcome of Depression International Network (ODIN) study reported prevalence of adjustment disorders in the general population, but solely for the subtype with depressive features, reporting a prevalence of $1 \%{ }^{8}$ Another study of the general population of Zurich over 65 years old reported a prevalence of $2.3 \% .^{9}$ In primary care, prevalence has been estimated to range from 1\% in France for the anxiety features subtype, ${ }^{10}$ to $18 \%$ for adjustment disorders overall in studies performed during the $1980 \mathrm{~s} .{ }^{5}$ Strain et al

†See editorial, pp. 90-92, this issue. reported a prevalence of $12 \%$ in hospital settings. ${ }^{11} \mathrm{~A}$ recent meta-analysis estimated that the prevalence of adjustment disorders was $19.4 \%$ in oncological and haematological settings and $15.4 \%$ in palliative care. ${ }^{12}$

Conceptually, adjustment disorders are an intermediate health condition between normal responses to stress and more severe emotional disorders such as anxiety and depression. ${ }^{2}$ A number of studies have found that adjustment disorders differ from mood disorders in some parameters. For instance, adjustment disorders were associated with more stressors, were more frequent in younger patients and less likely to occur in those living alone and in women. ${ }^{11,13}$ Runeson et al reported a shorter interval (1 month) between adjustment disorders and the emergence of suicidal behaviour than that found for depression ( 3 months) in young people. ${ }^{14}$ In contrast, other studies have failed to find differences between adjustment disorders and other psychiatric diagnoses. ${ }^{4,8}$ Moreover, there is scarce information on how general practitioners (GPs) manage this disorder, and the controversy deepens when we consider that the second edition of the International Classification of Primary Care (ICPC-2) makes no reference to adjustment disorders. ${ }^{15}$

Our study aims were to estimate the prevalence of adjustment disorders in primary care; to explore whether there are differences between primary care patients with adjustment disorders, those with mood or anxiety disorders and those with no mental disorder; and to describe recognition of adjustment disorders by GPs and the treatment given to patients with a standardised diagnosis of adjustment disorder. 


\section{Method}

The Diagnostic and Assessment Study of Mental Disorders in Primary Care (DASMAP) was a face-to-face, cross-sectional survey of a representative sample of adults (18 years or older) attending primary care health centres in Catalonia, one of the 17 regions or autonomous communities of Spain. ${ }^{16}$ Since 1981 these autonomous communities have been fully responsible for health and social care which is publicly financed, and near-universal coverage is provided. ${ }^{17}$ General practitioners have a key role in the recognition, diagnosis, treatment and referral of patients with mental disorders.

A stratified multistage probability sample without replacement was drawn. Replacement was prohibited to ensure that every individual had a known probability of selection. The sampling frame was the seven Catalonia health regions. The first stage consisted of the selection of primary care centres within each health region. A list of all centres and relevant data were obtained to enable the random selection. A previous filter of centres with fewer than 4000 attenders was done to exclude non-representative centres. The probability of selection of each centre was related to the population of the catchment area that it covered, so that centres with larger catchment areas were more likely to be selected. The number of centres selected in each region was proportional to the region's population. However, to ensure a minimum set of interviews even in the smaller regions, at least six centres were chosen per region. Of 352 centres, 77 participated in the DASMAP study. In the second stage, all GPs from the selected health centres were invited to participate. A total of 618 GPs agreed to take part. This represented nearly $69 \%$ of all GPs working at the 77 health centres. The third stage consisted of random selection of patients. A systematic sampling strategy was used, inviting every fifth patient from the daily appointment schedule of each participating GP. Of the 5402 patients pre-selected from the GP list, 654 did not keep their appointment, and 764 of the 4748 remaining patients declined to participate. A total of 169 patients were excluded because they showed cognitive impairment severe enough to preclude an adequate interview or they did not speak Spanish. The final sample comprised 3815 patients $(80.5 \%$ of those contacted). Further DASMAP study information is available elsewhere. ${ }^{16}$

\section{Measures}

Mental disorders were assessed with the Spanish versions of the Structured Clinical Interview for DSM-IV Axis I Disorders (SCID-I) (major depressive episode, dysthymic disorder, anxiety disorder modules and adjustment disorders excluding obsessivecompulsive disorder) and the Mini International Neuropsychiatric Interview (MINI; manic/hypomanic episodes, obsessive-compulsive disorder, substance and alcohol use disorders, anorexia nervosa and bulimia nervosa). ${ }^{18-20}$ Both instruments allow diagnoses according to DSM-IV criteria. One important DASMAP study objective was to assess the prevalence of mood and anxiety disorders in primary care in Catalonia, ${ }^{16}$ since such disorders were expected to be the most prevalent. Therefore, we chose the SCID-I because it is one of the most frequently used and reliable instruments for the assessment of these disorders. Since the complete SCID-I would have taken over an hour to administer, we decided to evaluate other mental disorders with a shorter instrument (the MINI).

Chronic medical conditions, defined by the WHO as 'health problems that require ongoing management over a period of years or decades, ${ }^{21}$ were assessed using a checklist including questions about a wide range of chronic physical conditions including asthma, bronchitis, ulcer, constipation, high blood pressure, heart disease, heart attack, stroke, epilepsy, migraine, allergies, arthritis, back pain, cancer, cataracts, diabetes, hearing impairment, neck pain, prostate-related conditions and vision impairment. Respondents were asked whether they had ever experienced any of the symptom-based checklist conditions. For conditions typically identified by medical diagnosis, respondents were asked whether a doctor or other health professional had ever told them they had the condition. Although checklist measures are imperfect, they provide useful information on both treated and currently untreated chronic conditions, ${ }^{22}$ and could predict healthcare use. $^{23}$ Moreover, self-report of chronic physical conditions shows moderate to high agreement with medical records. ${ }^{24}$

Quality of life was assessed with the Spanish version 2.0 of the 12-item Short Form Health Survey (SF-12). ${ }^{25,26}$ The SF-12 is a valid, reliable instrument for health-related quality of life assessment. Two measures were derived from the SF-12: a physical component summary scale (PCS-12) indicating physical quality of life, and a mental health summary scale (MCS-12) covering mental quality of life. Each scale uses all 12 items but with different weights. The PCS-12 and MCS-12 scales were scored using norm-based methods, with a mean of 50 and standard deviation of 10. Self-perceived disability (score 0-30), social support (0-100) and self-perceived stress $(0-10)$ were assessed through the Sheehan Disability Scales. ${ }^{27-29}$

Respondents were also asked about their main complaint: physical, emotional, administrative or a combination. In addition, they were asked about the number of visits to their GP in the previous 12 months. Psychotropic drug use was assessed using the information from the computerised clinical history. Regardless of the patients' diagnostic status, evaluators extracted information about any psychotropic medication prescribed to the participant in the previous 12 months, without distinguishing between occasional and long-term use. Psychotropic drugs were categorised according to the Anatomical Therapeutic Chemical (ATC) classifications, including anxiolytics (N05B), hypnotics and sedatives (N05C) and antidepressants (N06A). Diagnoses made by the GP were extracted from the clinical chart and recoded into a variable that included the presence or absence of adjustment disorders, other mental disorders or social problems. The GP diagnoses were based on ICD-9 or ICD-10, ${ }^{30,31}$ or on the ICPC-2. ${ }^{15}$

\section{Procedure}

A group of 20 trained psychologists evaluated the participants (training was provided by a panel of three experts and consisted of a 2-day course). Data were collected between October 2005 and March 2006 using a paper-and-pencil personal interview. After visiting their GP, individuals were invited to participate in the study. They were evaluated at the primary care centre after acceptance (signing an informed consent form). The instruments were administered during a clinical interview of approximately $45 \mathrm{~min}$. Following data collection, responses were processed using response automatic capture software TeleForm (Autonomy Cardiff, Vista, California, USA). Ethical approval was obtained from the Sant Joan de Déu Foundation ethics board.

\section{Statistical analysis}

Prevalence estimates of adjustment disorders and subtypes were expressed in both absolute numbers and weighted percentages with $95 \%$ confidence intervals. The results of the analysis were weighted to account for the varying probability of selection, given the stratified sampling. Three different logistic models were fitted 
to assess whether there were differences between adjustment disorders and major depression; between adjustment disorders and anxiety disorder; and between adjustment disorders and no mental disorder. In an additional analysis set we compared adjustment disorder plus depressive symptoms $v$. major depression, and adjustment disorder plus anxiety symptoms $v$. generalised anxiety disorder. Multilevel analyses were conducted considering primary care centre and GP as possible sources of random effects. The null models were statistically significant in all five comparisons for primary care centre, but in only one of the five comparisons for GP (adjustment disorder with depressive symptoms $v$. major depression). Thus, GP as a source of variability was ruled out in the analyses of the remaining four comparisons. Subsequently, we performed univariate multilevel logistic regression, including variables with significance $P \leqslant 0.2$ in univariate analysis in the final models.

Among those with adjustment disorders we estimated the percentage of cases recognised by the GP. The mean number of visits and the percentage of patients with a prescription of a psychotropic drug are described. We also tested multilevel analysis. Null models were not statistically significant, so we performed standard logistic regressions to ascertain which factors were associated with prescription among those with adjustment disorders. First, we tested bivariate models with psychotropic medication (yes/no) as the dependent variable and gender, age, type of adjustment disorder, disability, stress, social support, mental and physical quality of life, main reason for consultation, emotional problems and number of visits as the independent variables. In the final models we included variables with significance $P \leqslant 0.2$ in univariate analyses. The statistical analyses were conducted with Stata version 11 on Windows 7. All significance tests were made using two-sided tests evaluated at the $P<0.05$ level of significance.

\section{Results}

\section{Prevalence of adjustment disorder}

Of the 3815 study participants, $2.94 \%$ (95\% CI 2.21-3.91, $n=110$ ) met SCID-I criteria for a diagnosis of current adjustment disorder. Taking adjustment disorder subtypes into account, 29 of the 3815 participants presented with depressed $\operatorname{mood}(0.77 \%, 95 \%$ CI $0.49-1.21)$ and 50 had anxiety symptoms (1.34\%, 95\% CI $0.88-$ 2.02). The other people with adjustment disorder $(n=31$; $0.83 \%, 95 \%$ CI $0.54-1.28$ ) presented with another subtype (disturbance of conduct, unspecified or mixed symptoms). Table 1 shows the sociodemographic and clinical characteristics of the sample categorised by adjustment disorder subtypes and other disorders.

\section{Comparisons with depression and anxiety disorders and no mental disorder}

Table 2 shows the three final multivariate logistic models used to assess differences between adjustment disorder $v$. major depression; adjustment disorder $v$. anxiety disorder; adjustment disorder $v$. primary care patients with no mental disorder; adjustment disorder with depressive symptoms $v$. major depression; and adjustment disorder with anxiety symptoms $v$. generalised anxiety disorder.

\section{Adjustment disorders and major depression}

Adjustment disorders were less prevalent in women than major depressive disorder (odds ratio (OR) 0.36, 95\% CI 0.17-0.77). Compared with those with major depression, those with adjustment disorders were less likely to report emotional problems as a main reason for consultation $(\mathrm{OR}=0.24,95 \%$ CI $0.09-0.67)$. Those with adjustment disorders reported a better quality of life (both physical and emotional) than patients with major depression and had a better perception of social support. In addition, those with adjustment disorders were less likely to have been prescribed an antidepressant than those with major depression ( $\mathrm{OR}=0.43,95 \% \mathrm{CI} 0.19-0.98)$. When considering only the depressive subtype of adjustment disorder, we found that participants with this subtype scored more highly on mental quality of life (OR $=1.11,95 \%$ CI $1.00-1.24)$.

\section{Adjustment disorder and anxiety disorders}

In women, adjustment disorders were less prevalent than anxiety disorders $(\mathrm{OR}=0.42,95 \%$ CI $0.25-0.69)$. Patients with adjustment disorders also had higher mental quality of life scores $(\mathrm{OR}=1.02$, 95\% CI 1.00-1.04). Chronic medical illnesses were less likely in participants with adjustment disorders than in those with anxiety disorders $(\mathrm{OR}=0.45,95 \%$ CI $0.23-0.89)$, whereas participants with adjustment disorders had higher scores on self-perception of stress than those with anxiety disorders. Comparing the subtype of adjustment disorder plus anxiety with the generalised anxiety disorder group, we found that adjustment disorder with anxiety was less prevalent in women and showed higher scores for mental quality of life $(\mathrm{OR}=1.07,95 \% \mathrm{CI} 1.00-1.13)$. Prescription of antidepressants was also less likely in this subtype.

\section{Adjustment disorders and no mental disorder}

When comparing patients with adjustment disorders with primary care patients without mental disorders, we found that the former group had lower scores on mental quality of life than those with no mental disorder and also had higher levels of self-perceived stress. No other difference was found.

\section{Recognition and treatment of adjustment disorders in primary care}

\section{Recognition}

In only 2 of the 110 cases with a SCID-I diagnosis was 'adjustment disorder' written on the e-clinical chart (2\%, 95\% CI 0-4). In 17 of the 110 cases $(15 \%, 95 \%$ CI 9-22) the GP detected some kind of emotional problem or mental disorder. In 3 of the 110 cases the GP coded a social problem. In 72 of the 110 cases the GP recorded only physical problems on the chart $(65 \%, 95 \%$ CI 56-74). The remaining 16 cases $(14 \%, 95 \%$ CI $8-21)$ had incomplete charts. Citing emotional problems as the main reason for consulting was found for 14 of the 110 patients with adjustment disorders (13\%, 95\% CI 8-21).

\section{Use of services and treatment}

The mean number of visits in the previous 12 months was 5.6 (95\% CI 4.6-6.7). Regarding medication, 43 patients (37\%, 95\% CI $27-48 ; n=43)$ had at least one psychotropic prescription. Of these patients, $71 \%(95 \%$ CI 56-83; $n=32)$ had at least one anxiolytic, $10 \%(95 \%$ CI $4-24 ; n=4)$ at least one hypnotic-sedative and $45 \%(95 \%$ CI $31-60 ; n=18)$ at least one antidepressant. After adjusting for $P<0.2$ variables in the bivariate models, factors associated with psychotropic prescription among those with adjustment disorders were:

(a) mental quality of life (SF-12): decreasing probability of prescription as quality of life improves $(\mathrm{OR}=0.95,95 \% \mathrm{CI}$ $0.91-0.99)$

(b) emotional problems as the main reason for consultation $(\mathrm{OR}=6.19,95 \%$ CI 1.46-26.18); 
Table 1 Description of the samples

\begin{tabular}{|c|c|c|c|c|c|c|c|}
\hline & $\begin{array}{l}\text { Overall } \\
\text { AD } \\
n=110\end{array}$ & $\begin{array}{c}\text { AD with } \\
\text { depression } \\
n=29\end{array}$ & $\begin{array}{l}\text { AD with } \\
\text { anxiety } \\
n=50\end{array}$ & $\begin{array}{c}\text { Major depressive } \\
\text { episode } \\
n=339\end{array}$ & $\begin{array}{c}\text { Anxiety } \\
\text { disorder } \\
n=666\end{array}$ & $\begin{array}{c}\text { Generalised } \\
\text { anxiety disorder } \\
n=132\end{array}$ & $\begin{array}{l}\text { No mental } \\
\text { disorder } \\
n=2676\end{array}$ \\
\hline Female, \% & $60.3(49.2-70.5)$ & $65.3(46.0-80.6)$ & $52.3(35.5-64.9)$ & 79.1 (74.2-83.2) & $75.9(71.5-79.8)$ & 79.4 (71.4-85.5) & $59.2(56.9-61.4)$ \\
\hline Age, years: mean (range) & $52.0(47.9-56.1)$ & $57.6(50.4-64.8)$ & $50.6(44.2-57.1)$ & $45.2(38.5-52.0)$ & $50.6(48.9-52.2)$ & $50.6(48.0-53.1)$ & $56.1(55.1-57.1)$ \\
\hline \multicolumn{8}{|l|}{ Job status, \% } \\
\hline $\begin{array}{l}\text { Paid employment } \\
\text { Paid employment }\end{array}$ & $39.8(30.7-49.6)$ & $32.3(16.5-53.5)$ & $41.2(29.4-54.2)$ & $26.9(22.5-31.8)$ & $34.1(30.1-38.3)$ & $41.1(32.4-50.4)$ & $33.6(31.3-36.0)$ \\
\hline but on sick leave & $14.6(8.8-23.1)$ & $18.3(8.4-35.4)$ & $9.4(3.4-23.2)$ & $26.7(21.5-32.6)$ & $16.6(13.6-20.2)$ & $12.6(8.2-19.1)$ & $9.8(8.5-11.3)$ \\
\hline Other & $45.7(35.3-56.4)$ & $49.9(31.2-67.8)$ & $49.4(36.1-62.8)$ & $46.4(40.7-52.2)$ & $49.3(45.3-53.3)$ & $46.2(37.5-55.2)$ & $56.6(54.1-59.0)$ \\
\hline Chronic physical conditions, \% & $80.7(72.3-87.1)$ & $80.4(62.0-91.2)$ & $82.1(71.0-89.6)$ & $92.7(89.2-95.1)$ & $89.6(87.5-91.4)$ & $93.3(86.6-96.8)$ & $83.7(82.0-85.2)$ \\
\hline \multicolumn{8}{|l|}{$\begin{array}{l}\text { Assessment scale scores, } \\
\text { mean (range) }\end{array}$} \\
\hline Mental quality of life (SF-12) & $44.8(41.7-47.8)$ & $40.8(36.9-44.7)$ & $49.8(46.9-52.7)$ & $27.4(26.3-28.5)$ & $39.6(37.9-41.3)$ & $42.3(39.9-44.8)$ & $51.2(50.5-51.8)$ \\
\hline Physical quality of life (SF-12) & $46.2(43.3-49.0)$ & $42.7(37.4-48.0)$ & $45.1(41.8-48.4)$ & $40.5(38.9-42.0)$ & $44.5(43.5-45.6)$ & $45.9(43.9-47.9)$ & $46.2(45.6-46.7)$ \\
\hline Self-perceived disability & $8.0(5.7-10.3)$ & $9.1(5.1-13.0)$ & $6.4(3.5-9.2)$ & $16.5(15.4-17.7)$ & $10.4(9.3-11.4)$ & $7.5(6.0-9.1)$ & $4.9(4.5-5.4)$ \\
\hline Self-perceived stress & $5.4(4.4-6.3)$ & $5.4(4.0-6.8)$ & $5.3(4.0-6.6)$ & $6.7(6.2-7.3)$ & $4.8(4.4-5.2)$ & $4.7(4.1-5.3)$ & $2.4(2.1-2.6)$ \\
\hline Self-perceived social support & $81.2(76.3-86.0)$ & $81.6(70.8-92.3)$ & $83.2(75.2-91.1)$ & $63.8(59.2-68.3)$ & $73.3(70.2-76.4)$ & $72.3(66.7-77.9)$ & $80.6(78.7-82.5)$ \\
\hline $\begin{array}{l}\text { Mental/emotional problems } \\
\text { main reason for consultation, \% }\end{array}$ & $12.7(7.6-20.6)$ & $14.3(5.3-33.2)$ & $11.3(4.47-25.6)$ & $35.2(29.7-41.1)$ & $23.7(20.0-27.7)$ & $21.3(14.8-29.6)$ & $4.7(3.7-5.9)$ \\
\hline Number of visits, mean (range) & $5.6(4.6-6.7)$ & $5.9(4.4-7.4)$ & $5.6(3.9-7.5)$ & $9.9(8.6-11.3)$ & $7.6(6.9-8.3)$ & $6.5(5.4-7.6)$ & $5.9(5.6-6.3)$ \\
\hline \multicolumn{8}{|l|}{ Prescribed medication, \% } \\
\hline Anxiolytic & $26.4(12.5-36.2)$ & $24.0(12.4-41.2)$ & $25.9(14.4-42.2)$ & $47.9(43.0-53.0)$ & $37.5(32.4-42.9)$ & $48.9(39.0-58.9)$ & $15.6(14.0-17.3)$ \\
\hline Hypnotic sedative & $3.6(1.3-9.4)$ & $11.3(4.0-28.2)$ & 0 & $12.2(9.0-16.3)$ & $7.2(5.3-9.7)$ & $4.7(2.2-9.9)$ & $4.1(3.3-5.1)$ \\
\hline Antidepressant & $16.7(10.2-26.1)$ & $16.8(6.7-36.2)$ & $10.5(4.4-22.9)$ & $52.5(46.7-58.2)$ & $35.4(30.6-40.6)$ & $33.8(25.7-43.0)$ & $10.5(9.1-12.1)$ \\
\hline
\end{tabular}

(c) age: increasing probability of prescription with ageing $(\mathrm{OR}=1.04,95 \%$ CI 1.00-1.07);

(d) being employed but on sick leave $(\mathrm{OR}=4.47$, 95\% CI $1.02-$ 19.52).

\section{Discussion}

We found the prevalence of adjustment disorders in Catalan primary care to be $2.94 \%$, which is lower than expected and lower than in studies conducted over 20 years ago. When we compared our data with the most recent study by Semaan et al, focused on adjustment disorders with anxiety symptoms in primary care, rates were more similar - around $1 \% .^{10}$ Our data are similar to those reported in the general population such as the ODIN and Zurich studies. ${ }^{8,9}$ As others have shown, ${ }^{1-5,8}$ this low frequency could be because adjustment disorder, being an exclusion criterion, is a subordinate diagnosis. In fact, the SCID-I only asks about adjustment disorders once all others have been checked. ${ }^{18}$

Table 2 Comparisons between adjustment disorders and other mental disorders

\begin{tabular}{|c|c|c|c|c|c|}
\hline & \multicolumn{5}{|c|}{ Odds ratios $(95 \% \mathrm{Cl})^{\mathrm{a}}$} \\
\hline & $\begin{array}{c}\operatorname{AD}(1) \\
v . \operatorname{MDD}(0)\end{array}$ & $\begin{array}{l}\text { AD with depression } \\
\text { (1) v. MDD (0) }\end{array}$ & $\begin{array}{c}A D(1) \\
\text { v. anxiety (0) }\end{array}$ & $\begin{array}{l}\text { AD with anxiety } \\
\text { (1) } v . \text { GAD (0) }\end{array}$ & $\begin{array}{c}\text { AD (1) v. no mental } \\
\text { disorder(0) }\end{array}$ \\
\hline Women (reference men) & $0.36(0.17-0.77)^{\star *}$ & $0.38(0.06-2.13)$ & $0.42(0.25-0.69)^{* * *}$ & $0.27(0.09-0.78)^{*}$ & $0.68(0.43-1.06)$ \\
\hline Age & $1.02(0.99-1.05)$ & $1.02(0.97-1.07)$ & $1.01(0.99-1.03)$ & $1.01(0.98-1.04)$ & $1.00(0.99-1.01)$ \\
\hline $\begin{array}{l}\text { Paid employment but on sick leave } \\
\text { (reference paid employment) }\end{array}$ & $1.43(0.51-4.06)$ & & & & \\
\hline Other (reference paid employment) & $0.97(0.39-2.37)$ & & & & \\
\hline Mental quality of life (SF-12) & $1.13(1.09-1.17)^{* * *}$ & $1.11(1.00-1.24)^{\star}$ & $1.02(1.00-1.04)^{*}$ & $1.07(1.00-1.13)^{*}$ & $0.97(0.95-0.99)^{\star *}$ \\
\hline Physical quality of life (SF-12) & $1.07(1.04-1.12)^{* * *}$ & & & & \\
\hline Self-perceived disability & $0.97(0.92-1.02)$ & $0.88(0.76-1.00)$ & $0.98(0.95-1.01)$ & & $0.99(0.96-1.02)$ \\
\hline Self-perceived stress & $1.07(0.96-1.20)$ & $1.10(0.83-1.45)$ & $1.17(1.08-1.27)^{\star * *}$ & & $1.37(1.28-1.49)^{* * *}$ \\
\hline Self-perceived social support & $1.01(1.00-1.02)^{*}$ & $1.02(0.99-1.04)$ & $1.00(0.99-1.01)$ & $1.00(0.99-1.02)$ & \\
\hline Presence of chronic physical illness & $0.47(0.16-1.38)$ & & $0.45(0.23-0.89)^{*}$ & & \\
\hline Emotional problems as a main reason & $0.24(0.09-0.67)^{* *}$ & $0.43(0.05-3.76)$ & $0.60(0.29-1.25)$ & $1.69(0.34-8.30)$ & $1.79(0.80-4.00)$ \\
\hline Number of visits & $0.95(0.89-1.02)$ & & $0.97(0.93-1.01)$ & & \\
\hline Any anxiolytic or hypnotic sedative (N05) & $0.79(0.37-1.68)$ & $0.68(0.15-3.20)$ & $0.92(0.52-1.62)$ & $0.47(0.13-1.59)$ & $1.32(0.77-2.26)$ \\
\hline Any antidepressant (N06A) & $0.43(0.19-0.98)^{*}$ & $0.18(0.01-2.35)$ & $0.52(0.27-1.01)$ & $0.20(0.04-0.94)^{\star}$ & $0.93(0.46-1.91)$ \\
\hline PC centre variance & $1.06(0.27-4.08)$ & $0.32(0-224434.1)$ & $0.86(0.34-2.20)$ & $3.53(0.87-14.22)$ & $2.16(1.13-4.12)$ \\
\hline GP variance & & $5.18(0.31-121.42)$ & & & \\
\hline
\end{tabular}


Considering adjustment disorders as a 'residual' category could be an added complication and a gap between theory and practice: the clinician knows that the symptoms will resolve quickly, but as the five-symptom threshold has been reached an adjustment disorder diagnosis cannot be made. From a clinical point of view, giving different diagnoses to two patients coping with a stressful situation because one had four symptoms (feeling sad most of the day, decrease in appetite, insomnia and fatigue) and the other five (same symptoms plus agitation) is not helpful. ${ }^{6}$ Solutions to this could include eliminating adjustment disorders from DSM-5 or returning to the old classification regarding reactive/endogenous depression, or even creating a new stress response category. ${ }^{7}$

\section{Comparison with other mental disorders}

Our study showed adjustment disorder to be an intermediate category between absence of mental disorder and the meta-category of major depression/anxiety disorders. The condition has a distinctive profile: better than patients with either depression or anxiety, but worse than patients with no mental disorder. The same pattern is observed in comparison of specific subgroups: the main difference between adjustment disorders with depressive features and major depressive disorder is quality of life (better mental quality of life for those with adjustment disorders plus depressive symptoms), and the same is true for adjustment disorders with anxiety and generalised anxiety disorder. Nevertheless, the small sample size could explain why other differences were not found. Compared with those with major depression or anxiety disorder, adjustment disorders were less likely in women. This is consistent with other papers, ${ }^{11,13}$ and could suggest they are different conditions. Another interesting finding is that patients with adjustment disorders reported high levels of self-perceived stress, even higher than those with an anxiety disorder. Although we did not assess specific stressors, the fact that patients with adjustment disorders perceive themselves as more stressed could support the Strain \& Friedman idea of considering adjustment disorders as a stress response syndrome. ${ }^{7}$

\section{Recognition and treatment}

Specific recognition of adjustment disorders as a diagnostic entity by the GP is also low, although it seems that some kind of psychological or emotional distress is recognised. Among reasons for this, we should bear in mind that an adjustment disorder category is not present in the ICPC-2 and that only slightly less than $13 \%$ of people with adjustment disorders cited emotional problems as the main reason for consultation, which is known to be the main factor associated with mental disorder recognition by GPs. ${ }^{32}$ In fact, one adjustment disorder criterion is related to marked distress that is greater than expected, which may be difficult to evaluate. General practitioners, aware that the distress is associated with a specific and temporary stressor, would prefer to monitor rather than label the patient. Moreover, we are considering the psychiatric point of view as a gold standard for GP activity and this may be inappropriate.

Regarding treatment, $37 \%$ of patients with adjustment disorders had a psychotropic drug prescribed; this is lower than previously reported. ${ }^{10}$ Little evidence about treatment of adjustment disorders is available, but studies suggest that psychological interventions are more effective than drug therapy, which is not recommended. ${ }^{1-5}$ However, the fact that anxiolytics were the most prescribed drug leads us to consider whether GPs are prescribing them to treat insomnia or as a point treatment for occasional high levels of anxiety. Unfortunately, this information was not available so we cannot determine prescription reasons. It is interesting that those with adjustment disorders also had a lower probability of antidepressant prescription (when compared with those with major depression), which could be seen as a proxy for severity. Nevertheless, our data suggest that there is a misuse of antidepressants in patients who could benefit from psychological treatment or just watchful waiting.

\section{Strengths and limitations}

A strength of this study is that it was conducted among a large, representative sample of people attending primary care. Moreover, few studies have been performed with such a large quantity of epidemiological data in primary care that also studied adjustment disorders using SCID-I. Given the revisions of DSM-IV and ICD-10, this is a critical moment for the presentation of data concerning adjustment disorders. However, some limitations should be mentioned. First, we did not specifically assess stressful life events but rather assessed adjustment disorders if participants spontaneously reported a stressful event in the previous months. This may have underestimated prevalence. Nevertheless, those who spontaneously reported a stressful event would be considered as more disabled, assuming we are not medicalising normal stress reactions. ${ }^{2}$ Finally, self-reporting of chronic illness may have complicated the assessment.

\section{Implications}

In conclusion, our data suggest that adjustment disorders, as currently defined by the DSM, have a distinct profile that could be useful in classifying patients. Patients with adjustment disorders are less disabled but more stressed than those with $\operatorname{mood} /$ anxiety disorders. However, more debate and research is needed to determine whether the current definition is the most clinically useful conceptualisation.

Anna Fernández, PhD, Research and Development Unit, Parc Sanitari Sant Joan de Déu, Fundació Sant Joan de Déu, Red de Investigación en Actividades Preventivas Promoción de la Salud en Atención Primaria (RedIAPP), Instituto de Salud Carlos III, and Clinical and Health Psychology Department, Universitat Autònoma de Barcelona, Barcelona; Juan M. Mendive, MD, Centro de Salud La Mina, Institut Català de la Salut, Sant Adrià de Besos, Barcelona; Luis Salvador-Carulla, MD, Research and Development Unit, Parc Sanitari Sant Joan de Déu, Fundació Sant Joan de Déu, and RedIAPP, Instituto de Salud Carlos III, Barcelona; María Rubio-Valera, MSC, RedIAPP, RedlAPP, Instituto de Salud Carlos III, Barcelona; Maria Rubio-Valera, MSC, RedIA
Instituto de Salud Carlos III, Barcelona; Juan Vicente Luciano, PhD, Alejandra Pinto-Meza, PhD, Research and Development Unit, Parc Sanitari Sant Joan de Déu, Fundació Sant Joan de Déu, and RedIAPP, Instituto de Salud Carlos III, Barcelona; Josep Maria Haro, MD, Research and Development Unit, Parc Sanitari Sant Joan de Déu, Fundació Sant Joan de Déu, and Centro de Investigación Biomédica en Red de Salud Mental (CIBERSAM), Instituto de Salud Carlos III, Barcelona; Diego J. Palao, MD, Centre de Salut Mental, Corporació Sanitaria Parc Taulí, Institut Universitari Fundació Parc Taulí-Universitat Autònoma de Barcelona, Sabadell; Juan A. Bellón MD, RedIAPP, Instituto de Salud Carlos III, Barcelona, and Centro de Salud El Palo Unidad de Investigación del Distrito de Atención Primaria de Málaga, Departamento de Medicina Preventiva y Salud Pública, Universidad de Málaga; Antoni SerranoBlanco, MD, Research and Development Unit, Parc Sanitari Sant Joan de Déu, Fundació Sant Joan de Déu, and RedIAPP, Instituto de Salud Carlos III, Barcelona, Spain; the DASMAP investigators

Correspondence: Dr Anna Fernández, Parc Sanitari Sant Joan de Déu, Research and Development Unit, Fundació Sant Joan de Déu, Dr Antoni Pujadas 42 08830 Sant Boi de Llobregat, Barcelona, Spain. Email: afernandez@pssjd.org

First received 5 May 2011, final revision 9 Feb 2012, accepted 15 Mar 2012

\section{Funding}

The study was funded by the Direcció General de Planificació i Avaluació Sanitària, Departament de Salut, Generalitat de Catalunya, Barcelona. A.F. and J.V.L. are grateful to Departament de Salut, Generalitat de Catalunya, Barcelona. A.F. and J.V.L. are grateful to
the Ministerio de Sanidad y Consumo, Instituto de Salud Carlos III (Red RD06/0018/0017) for a pre-doctoral and a post-doctoral contract respectively. 


\section{Acknowledgements}

We are grateful to Sir David Goldberg for his helpful comments on a draft version of this manuscript. We also thank Stephen Kelly for his help in English language editing.

\section{References}

1 Semprini F, Fava GA, Sonino N. The spectrum of adjustment disorders: too broad to be clinically helpful. CNS Spectr 2010; 5: 382-8.

2 Baumeister H, Maercker A, Casey P. Adjustment disorders with depressed mood. A critique of its DSM-IV and ICD-10 conceptualisations and recommendations for the future. Psychopathology 2009; 42: 139-47.

3 Baumeister $\mathrm{H}$, Kufner $\mathrm{K}$. It is time to adjust the adjustment disorder category. Curr Opin Psychiatry 2009; 22: 409-12.

4 Carta MG, Blestrieri M, Murru A, Hardoy MC. Adjustment disorder: epidemiology, diagnosis and treatment. Clin Pract Epidemiol Ment Health 2009; 5: 15

5 Casey P. Adjustment disorder: epidemiology, diagnosis and treatment. CNS Drugs 2009; 23: 927-38.

6 Casey P, Dowrick C, Wilkinson G. Adjustment disorders. Fault line in the psychiatric glossary. Br J Psychiatry 2001; 179: 479-81.

7 Strain JJ, Friedman MJ. Considering adjustment disorders as stress response syndromes for DSM-5. Depress Anxiety 2011; 28: 818-23.

8 Casey P, Maracy M, Kelly BD, Lehtinen V, Ayuso-Mateos JL, Dalgard OS, et al. Can adjustment disorder and depressive disorder be distinguished? Results from ODIN. J Affect Disord 2006; 92: 291-7.

9 Maercker A, Forstmeier S, Enzler A, Krüsi G, Hörler E, Maier C, et al Adjustment disorders, posttraumatic disorder, and depressive disorders in old age: findings from a community survey. Compr Psychiatry 2008; 49: $113-20$

10 Semaan W, Hergueta $T$, Bloch J, Charpak $Y$, Duburcq A, Le Guern ME, et al. Cross-sectional study of the prevalence of adjustment disorder with anxiety in general practice [in French]. Encephale 2001; 27: 238-44.

11 Strain JJ, Smith GC, Hammer JS, McKenzie DP, Blumenfield MB, Muskin P, et al. Adjustment disorder: a multisite study of its utilization and interventions in the consultation-liaison psychiatry settings. Gen Hosp Psychiatry 1998; 20: 139-49.

12 Mitchell AJ, Chan M, Bhatti H, Halton M, Grassi L, Johansen C, et al. Prevalence of depression, anxiety, and adjustment in oncological, haematological, and palliative-care settings: a meta-analysis of 94 interview-based studies. Lancet Oncol 2011; 12: 160-74.

13 Snyder S, Strain JJ, Wolf D. Differentiating major depression from adjustmen disorders with depressed mood in the medical setting. Gen Hosp Psychiatry 1990; 12: 159-65.

14 Runeson BS, Beskow J, Waern M. The suicidal process in suicides among young people. Acta Psychiatr Scand 1996; 93: 35-42.

15 World Health Organization. International Classification of Primary Care, 2nd edn. WHO, 2003.

16 Serrano-Blanco A, Palao DJ, Luciano JV, Pinto-Meza A, Lujan L, Fernández A et al. Prevalence of mental disorders in primary care: results from the diagnosis and treatment of mental disorders in primary care study (DASMAP). Soc Psychiatry Psychiatr Epidemiol 2010; 45: 201-10.

17 Salvador L, Garrido M, McDaid D, Haro JM. Financing mental health care in Spain: context and critical issues. Eur Psychiatry 2006; 20: 29-44.

18 First MB, Gibbon M, Spitzer RL, Williams JBW. User's Guide for the Structured Clinical Interview for DSM-IV Axis I Disorders. Research Version. Biometrics Research, 2002.

19 Sheehan DV, Lecrubier $Y$, Sheehan $H$, Amorim $P$, Janavs J, Weiller $E$, et al The Mini-International Neuropsychiatric Interview (M.I.N.I.): the development and validation of a structured diagnostic psychiatric interview for DSM-IV and ICD-10. J Clin Psychiatry 1998; 59: 22-33.

20 Ferrando L, Franco AL, Soto M, Bobes J, Soto O, Franco L, et al. MINI International Neuropsychiatric Interview. (Spanish version 5.0.0.) DSM-IV Instituto IAP, 1998.

21 World Health Organization. Innovative Care for Chronic Conditions. WHO, 2002

22 Knight M, Stewart-Brown S, Fletcher L. Estimating health needs: the impact of a checklist of conditions and quality of life measurements on health information derived from community surveys. J Public Health Med 2001; 23: $179-86$

23 Fan VS, Au D, Heagerty P, McDonell MB, Deyo RA, Fihn SD. Validation of case-mix measures derived from self-reports of diagnoses and health. J Clin Epidemiol 2002; 55: 371-80.

24 National Center for Health Statistics. Evaluation of National Health Interview Survey Diagnostic Reporting. Vital Health Stat Series 2 (120). NCHS, 1994

25 Gandek B, Ware JE, Aaronson NK, Apolone G, Bjorner JB, Brazier JE, et al. Cross-validation of item selection and scoring for the SF-12 Health Survey in nine countries: results from the IQOLA Project. International Quality of Life Assessment. J Clin Epidemiol 1998; 51: 1171-8.

26 Vilagut G, Ferrer M, Rajmil L, Rebollo P, Permanyer-Miralda G, Quintana JM, et al. The Spanish version of the Short Form 36 Health Survey: a decade of experience and new developments. Gac Sanit 2005; 19: 135-50.

27 Bobes J, Badia X, Luque A, García M, González MP, Dal-Re R. Validation of the Spanish version of the Liebowitz Social Anxiety Scale, Social Anxiety and Distress Scale and Sheehan Disability Inventory for the evaluation of social phobia [in Spanish]. Med Clin (Barc) 1999; 112: 530-8.

28 Sheehan DV, Harnett-Sheehan K, Raj BA. The measurement of disability. Int J Clin Psychopharmacol 1996; 11: 89-95.

29 Luciano JV, Bertsch,J, Salvador-Carulla L, Fernández A, Pinto-Meza A, Haro $\mathrm{JM}$, et al. Factor structure, internal consistency and construct validity of the Sheehan Disability Scale in a Spanish primary care sample. J Eval Clin Pract 2010; 16: 895-901.

30 World Health Organization. International Statistical Classification of Diseases and Related Health Problems (ICD-9). WHO, 1978

31 World Health Organization. International Statistical Classification of Diseases and Related Health Problems (ICD-10). WHO, 1992.

32 Fernández A, Pinto-Meza A, Bellón JA, Roura-Poch P, Haro JM, Autonell J, et al. Is major depression adequately diagnosed and treated by general practitioners? Results from an epidemiological study. Gen Hosp Psychiatry 2010; 32: 201-9. 